\title{
Perpendicular Magnetic Anisotropy in CoFeB/Pd Bilayers
}

\author{
Ciarán Fowley, Nicolas Decorde, Kaan Oguz, Karsten Rode, Huseyin Kurt, and J. M. D. Coey \\ CRANN, School of Physics, Trinity College, Dublin 2, Ireland
}

\begin{abstract}
Perpendicular magnetic anisotropy is observed in ultrathin $(\sim 0.6 \mathrm{~nm})$ amorphous $\mathrm{Co}_{40} \mathrm{Fe}_{40} \mathrm{~B}_{20}$ when sputtered on an $\mathrm{MgO}(001)$ buffer layer and capped with Pd. The layers are superparamagnetic with a blocking temperature of $\sim 230 \mathrm{~K}$, below which they show an exponential temperature dependence of coercivity. Perpendicular magnetic anisotropy is observed in the as-deposited state and the mechanism is different from that of $\mathrm{CoFeB/Pt}$, which requires postannealing. These ultrathin layers could be a model system for studies of electric field effects on magnetic anisotropy.
\end{abstract}

Index Terms-Perpendicular magnetic anisotropy (PMA), thin-film capacitors.

\section{INTRODUCTION}

$\mathbf{R}$ ECENTLY, there has been intense interest in ultrathin films with perpendicular magnetic anisotropy (PMA) to reduce the critical current density $j_{c}$ for spin transfer torque (STT) switching [1] and to study the effect of an electric field on magnetic anisotropy [2]. In spin-valve devices, such as giant magnetoresistance (GMR) or tunneling magnetoresistance (TMR) nanopillars, the use of electrodes exhibiting PMA can increase the spin transfer torque efficiency and, hence, reduce $j_{c}$ [3].

PMA can be easily detected in bilayer systems by magneto-optical Kerr effect (MOKE) and extraordinary Hall effect (EHE). Bilayer films are also ideal systems for studying the electric-field control of magnetic properties [2], [4], [5]. Another advantage is the fact that the exhibited PMA is very sensitive to layer thickness and sample structure. Weisheit $e t$ al. demonstrated a change in the coercivities of $\mathrm{FePt}$ and $\mathrm{FePd}$ $\mathrm{L} 1_{0}$-ordered alloys upon application of an electric field in an electrolytic capacitor [2]. Maruyama et al. have demonstrated a $\sim 40 \%$ change in magnetocrystalline anisotropy in epitaxial ultrathin $\mathrm{Fe}(001)$ [4], and more recently, electric-field-assisted magnetization reversal in $\mathrm{FeCo}$ layers [5]. In both cases, the electric field was applied across a micron-thick polyimide layer. The effect originates from the fact that the filling of $3 d$ orbitals in the ferromagnetic layer is affected by the application of an electric field [4]. So far, the modification of anisotropy has only been investigated by MOKE.

For an electric field to have a measureable effect on the magnetization of a ferromagnetic metal, the ferromagnetic layer should be only a few monolayers thick due to the screening effect of conduction electrons. PMA layers are good candidates for electric-field effect studies since the anisotropy is realized for layers that are only a few monolayers thick. Most bilayers exhibiting PMA require a sufficiently thick metallic seed layer. Examples of these systems include $\mathrm{Co} / \mathrm{Pt}$ and $\mathrm{Co} / \mathrm{Pd}$ multilayers [6], [7]. In order to build a capacitor structure for the study of the electric-field modification of anisotropy, the ferromagnetic layer must be deposited on top of or beneath an insulating

Manuscript received October 31, 2009; revised January 16, 2010; accepted February 21, 2010. Current version published May 19, 2010. Corresponding author: C. Fowley (e-mail: fowleyc@tcd.ie).

Color versions of one or more of the figures in this paper are available online at http://ieeexplore.ieee.org.

Digital Object Identifier 10.1109/TMAG.2010.2044374 layer. In the normal case of a $\mathrm{Pt} / \mathrm{Co} / \mathrm{Pt}$ (or $\mathrm{Pd} / \mathrm{Co} / \mathrm{Pd}$ ) sandwich, both $\mathrm{Pt}(\mathrm{Pd})$ layers induce a strong interfacial anisotropy which serves to rotate and stabilize the magnetization of the Co layer out of plane. It is generally agreed that this is due to magnetocrystalline and magnetoelastic anisotropies at the $\mathrm{Co} / \mathrm{Pt}$ and $\mathrm{Co} / \mathrm{Pd}$ interfaces [8], [9]. The requirement for a solid-state capacitor structure is that one metal interface must be replaced with an insulator, such as $\mathrm{MgO}$ or $\mathrm{AlO}_{\mathrm{x}}$. These layers do not induce a strong interfacial anisotropy, like Pt or $\mathrm{Pd}$, in the as-deposited state. Nevertheless, Nistor et al. recently demonstrated that it is possible to stabilize PMA in thin layers of $\mathrm{CoFeB}$ grown on a buffer layer of $\mathrm{MgO}$ capped by $\mathrm{Pt}$ after annealing at $350{ }^{\circ} \mathrm{C}$ [10]. It has also been shown that with high temperature annealing, it is possible to stabilize PMA with $\mathrm{AlO}_{\mathrm{x}}$ underlayers and this is attributed to the hybridization of the $\mathrm{Co}$ and $\mathrm{O}$ orbitals after high-temperature annealing [11].

In previous studies, it has also been shown that $\mathrm{CoFeB}$ layers grown on $\mathrm{MgO}$ with thicknesses below $1 \mathrm{~nm}$ are superparamagnetic [12], [13]. Ultrathin polycrystalline metallic layers can form islands on a surface if the roughness and surface wetting are not optimal, which can increase the minimum thickness required for a continuous film. However, amorphous alloys, such as $\mathrm{CoFeB}$, can form continuous layers at smaller thicknesses.

Here, we present results on an ultrathin layer $(0.6 \mathrm{~nm})$ of amorphous $\mathrm{CoFeB}$ exhibiting PMA on an $\mathrm{MgO}$ buffer in the as-deposited state when capped with Pd. This system offers an opportunity to study changes in magnetic anisotropy under the application of an electric field by using EHE.

\section{EXPERIMENTAL METHOD}

Multilayer samples were grown with the following structure: $\mathrm{Si} / \mathrm{SiO}_{2} /$ underlayer/FM/cap . The underlayers used were Ta $5 / \mathrm{Pd} 5$ and $\mathrm{MgO} 1.4$ (thicknesses in nanometers). FM layers were $\mathrm{Co}$ and $\mathrm{Co}_{40} \mathrm{Fe}_{40} \mathrm{~B}_{20}$. Cap layers were Pd and Pt.

All samples were deposited on 1 " square thermally oxidized $\mathrm{Si}(100)$ wafers using a fully automated SFI Shamrock deposition tool. Samples were processed without breaking vacuum. $\mathrm{CoFeB}, \mathrm{Pt}, \mathrm{Pd}$, and Ta were dc-sputtered at an Ar pressure of 3 mTorr in a chamber with a base pressure of less than $2 \times$ $10^{-7}$ Torr . MgO was deposited by radio-frequency sputtering using argon in a target-facing-target configuration at a pressure of 4 mTorr in a chamber with a base pressure of less than $1 \times 10^{-8}$ Torr .

Magnetization measurements were performed in a Quantum Design Superconducting Quantum Interference Device (SQUID) magnetometer. Low-temperature measurements 


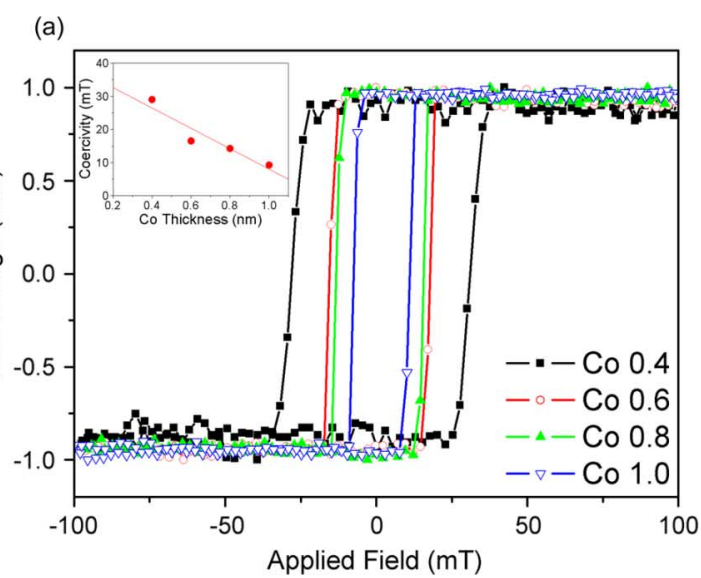

(b)

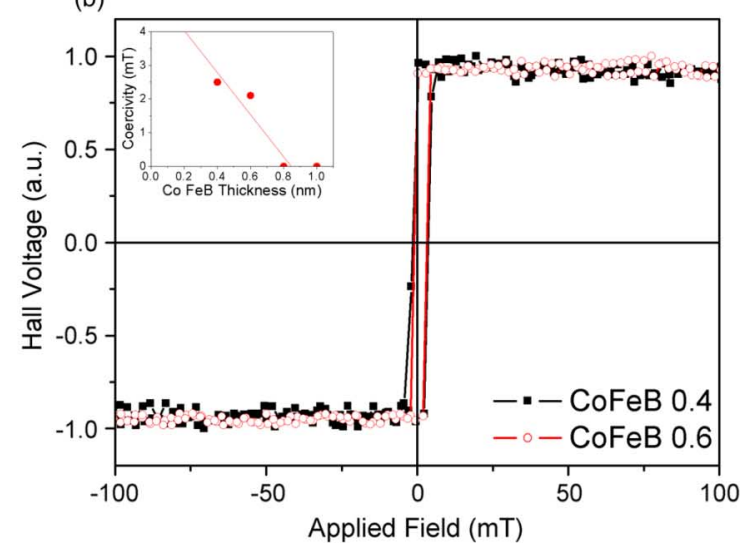

Fig. 1. FM layer thickness dependence for $\mathrm{Ta} / \mathrm{Pd} / \mathrm{FM} / \mathrm{Pd}$ sandwiches for (a) $\mathrm{Co}$ and (b) CoFeB. Insets: coercivity as a function of FM layer thickness.

were carried out in a cryostat with a base temperature of $13 \mathrm{~K}$ within an electromagnet capable of generating fields up to 180 mT. X-ray diffraction was carried out in a Philips X'Pert Pro diffractometer with an $X^{\prime}$ Celerator module.

\section{RESULTS AND DISCUSSION}

We first investigated the perpendicular magnetic anisotropy in $\mathrm{Pd} / \mathrm{Co} / \mathrm{Pd}$ and $\mathrm{Pd} / \mathrm{CoFeB} / \mathrm{Pd}$ sandwiches at room temperature. As the CoFeB alloy contains $20 \%$ boron, the alloy is expected to be amorphous regardless of substrate (in this case, $\mathrm{Pd}$ ) [14].

The results are shown in Fig. 1. The coercivity decreases with magnetic layer thickness, which is consistent with the competition between the volume anisotropy term, $K_{V}$ and the surface anisotropy term $K_{\mathrm{S}}$. In the case of Co, the PMA exists up to a Co thickness of $1.0 \mathrm{~nm}$, but in the case of $\mathrm{CoFeB}$, both $\mathrm{H}_{\mathrm{c}}$ and the thickness range of PMA are reduced. For the thinnest magnetic layers $(0.4 \mathrm{~nm}), \mathrm{H}_{\mathrm{c}}$ is $40 \mathrm{mT}$ in the case of Co and 4 $\mathrm{mT}$ in the case of $\mathrm{CoFeB}$. The order of magnitude difference is similar to [10].

After investigating the PMA in the $\mathrm{Pd} / \mathrm{CoFeB} / \mathrm{Pd}$ system, we replaced the $\mathrm{Ta} / \mathrm{Pd}$ underlayer with $\mathrm{MgO}$. In the following structure $\mathrm{Si} / \mathrm{SiO}_{2} / \mathrm{MgO} / \mathrm{CoFeB} / \mathrm{Pd}$, we varied the $\mathrm{CoFeB}$ thickness and examined the EHE response. We also investigated the crystal structure of the $\mathrm{MgO}$ and Pd layers.

Fig. 2(a) shows the EHE response as a function of CoFeB layer thickness. At 0.5 and $0.6 \mathrm{~nm}$, we see a vertical jump around zero field in the EHE measurements, indicative of a perpendicular easy axis of magnetization. At 0.4 and $0.7 \mathrm{~nm}$, we observe
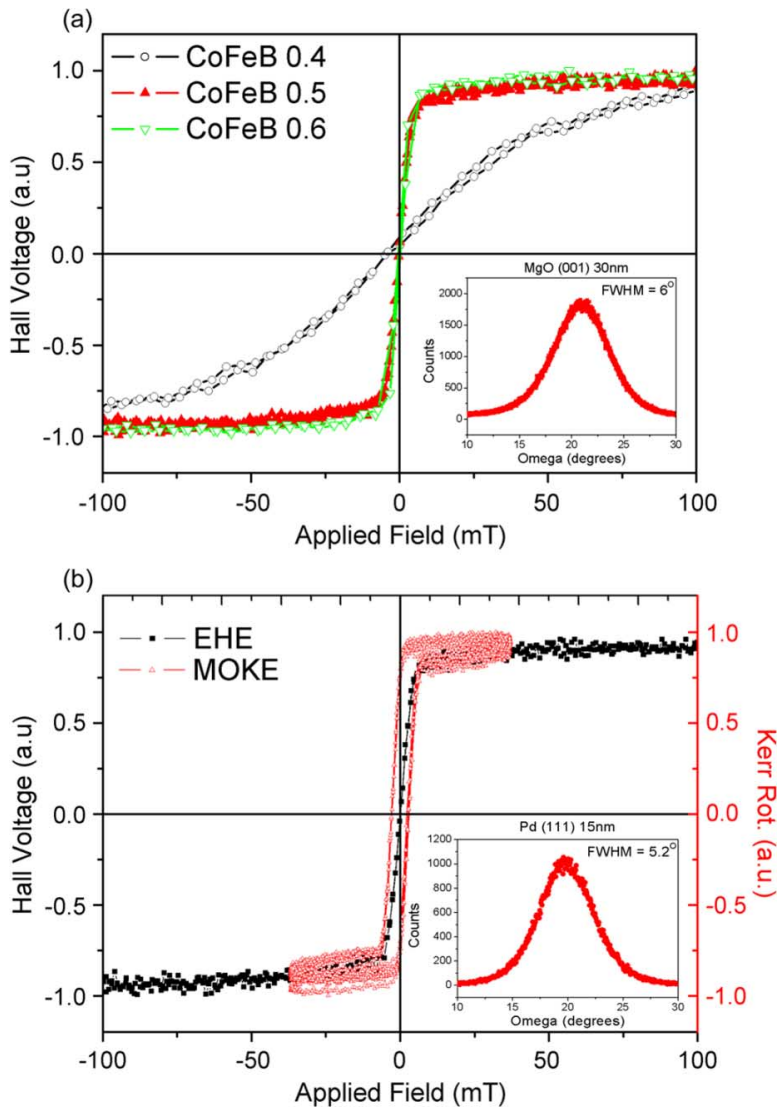

Fig. 2. (a) EHE response of $\mathrm{MgO} 1.4 / \mathrm{CoFeB} / \mathrm{Pd} 5$ as the thickness of $\mathrm{CoFeB}$ is varied. Inset: rocking curve for thick $(30 \mathrm{~nm}) \mathrm{MgO}$ underlayer. (b) MOKE and $\mathrm{EHE}$ loop comparison for $\mathrm{MgO}$ 1.4/CoFeB 0.6/Pd 5. Inset: rocking curve for the thick (15 nm) Pd capping layer.

a linear EHE response. Since the coercivity is zero for all thicknesses and there is no remanence, we think the $\mathrm{CoFeB}$ layer to be superparamagnetic on $\mathrm{MgO}$ at all thicknesses investigated [15]. Fig. 2(b) shows EHE and Kerr loops for the 0.6-nm film. The MOKE loop shows coercivity, implying that the magnetization is blocked on the $\sim$ ms time scale. The full EHE loop by comparison takes several minutes to complete.

To investigate the crystal structure of $\mathrm{MgO}$, we grew samples with a thick underlayer $(30 \mathrm{~nm})$ for XRD measurements. A weak (001) texture was observed from the rocking curve (we deposited the same $\mathrm{CoFeB} / \mathrm{Pd}$ structure on top and found no qualitative difference in EHE response between the differing $\mathrm{MgO}$ thicknesses) see Fig. 2(a) inset. From the rocking curve FWHM data, using Scherrer's formula, we calculate the average (001) grain size to be $9.6 \mathrm{~nm}$. The (001) texture of $\mathrm{MgO}$ implies that the $\mathrm{CoFeB}$ layer grows amorphous as seen in the case of $\mathrm{MgO}$ magnetic tunnel junctions [16]. Similarly, to investigate the crystal structure of the Pd layer, we deposited a sample with a thick Pd layer (15 nm) on top of the $\mathrm{MgO} 1.4 / \mathrm{CoFeB} 0.6$ structure and examined it by XRD. A (111) texture was observed from the rocking curve which is consistent with the appearance of PMA in the system, see Fig. 2(b) inset. As in the case of the thick $\mathrm{MgO}$ layer, we calculated a crystallite size of $11 \mathrm{~nm}$ for $\mathrm{Pd}$ from the rocking curve FWHM data. Again, no qualitative difference in EHE response between the differing Pd thicknesses was found. 

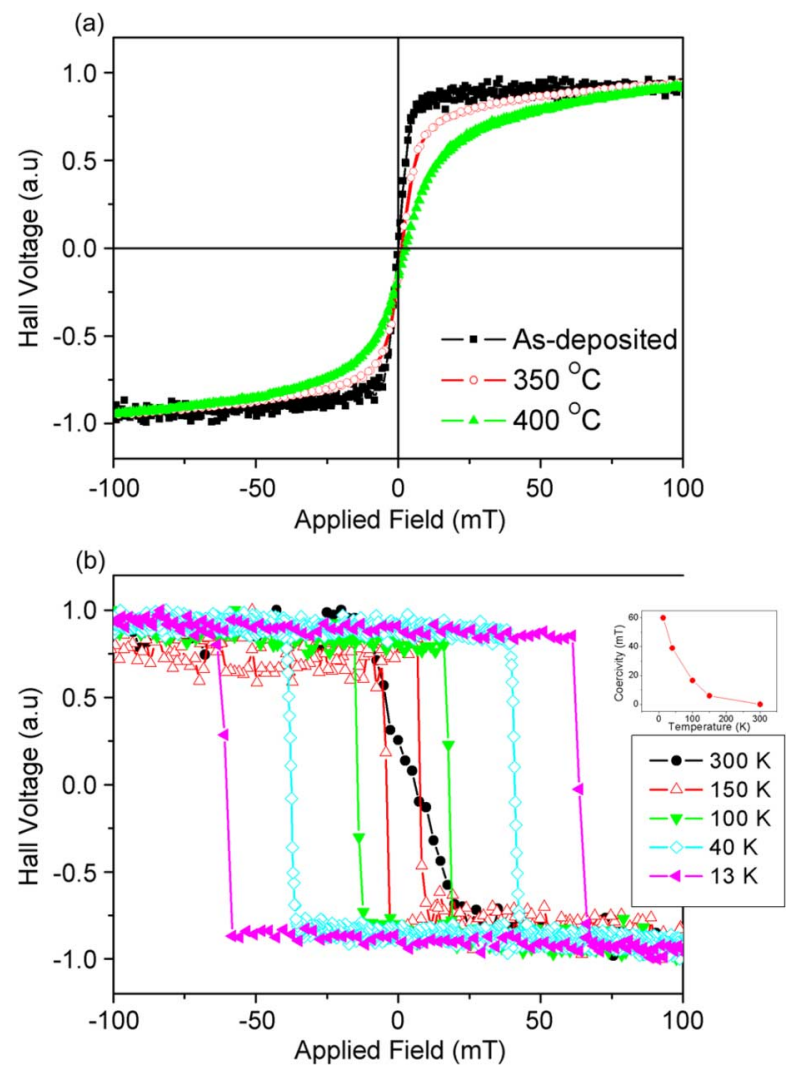

Fig. 3. (a) EHE response of $\mathrm{MgO} 1.4 / \mathrm{CoFeB} 0.6 / \mathrm{Pd} 5$ after high temperature annealing. No stabilization of the PMA was observed. (b) Low-temperature EHE measurements of unannealed $\mathrm{MgO} 1.4 / \mathrm{CoFeB} 0.6 / \mathrm{Pd} 5$. The film becomes ferromagnetic at approximately $228 \mathrm{~K}$.

Since perpendicular anisotropy was enhanced due to optimum oxidation of the $\mathrm{O}_{\mathrm{x}} / \mathrm{Co}$ interface in the case of [10] and [11], we investigated the effect of high-temperature annealing on the $\mathrm{MgO} / \mathrm{CoFeB} / \mathrm{Pd}$ samples. We were still able to get a response from the EHE measurements with annealing temperatures up to $400{ }^{\circ} \mathrm{C}$ [Fig. 3(a)]. However, we did not see an enhancement of perpendicular anisotropy as a function of temperature in contradiction with the $\mathrm{MgO} / \mathrm{CoFeB} / \mathrm{Pt}$ case.

We investigated the superparamagnetic behavior by conducting low-temperature measurements on the $\mathrm{MgO} 1.4 / \mathrm{CoFeB}$ $0.6 / \mathrm{Pd} 5$ sample. Below approximately $230 \mathrm{~K}$, the sample is blocked and exhibits ferromagnetic behavior with $\mathrm{H}_{\mathrm{c}}$ increasing exponentially as the temperature is decreased. This is shown in Fig. 3(b) and the inset. SQUID data (not shown) suggest the perpendicular axis is the easy axis at room temperature. The saturation magnetization of the $\mathrm{CoFeB}$ layer was determined from SQUID measurements to be $1.0 \mathrm{MA} / \mathrm{m}$.

We have also reproduced (by sputtering) perpendicular anisotropy upon annealing the $\mathrm{MgO} / \mathrm{CoFeB} / \mathrm{Pt}$ as observed in [10]. The high-temperature response is different from that of $\mathrm{MgO} / \mathrm{CoFeB} / \mathrm{Pd}$. This might be due to different migration characteristics of B with Pt or Pd capping.

As a final step, we investigated the effect of the application of an electric field on the unannealed $\mathrm{MgO} 1.4 / \mathrm{CoFeB} 0.6 / \mathrm{Pd} 5$ system. A small but detectable change of coercivity was found; the change in $\mathrm{H}_{\mathrm{c}}$ was $0.3 \%$ in an applied field of $250 \mathrm{MV} / \mathrm{m}$. There was no detectable change in magnetization perpendicular to the plane according to the EHE measurement.

\section{CONCLUSION}

Clear evidence is found for perpendicular magnetic anisotropy in the $\mathrm{Pd} / \mathrm{CoFeB} / \mathrm{Pd}$ bilayer system up to a $\mathrm{CoFeB}$ thickness of $0.6 \mathrm{~nm}$. However, in the sputtered $\mathrm{MgO} / \mathrm{CoFeB} / \mathrm{Pd}$ system, where the $\mathrm{CoFeB}$ layer is amorphous, it may be superparamagnetic at room temperature despite the fact that it is a continuous film [15]. High-temperature annealing does not enhance the perpendicular anisotropy, which is not the case in the $\mathrm{MgO} / \mathrm{CoFeB} / \mathrm{Pt}$ system. By cooling below the blocking temperature $(\sim 230 \mathrm{~K})$, it is possible to achieve coercivity, and a small effect of applied electric field on the coercivity of $\mathrm{CoFeB}$ was measured.

\section{ACKNOWLEDGMENT}

The authors would like to thank Dr. M. Venkatesan for SQUID measurements. This work was supported by Science Foundation Ireland under the MANSE contract 05/IN/1850. It was also conducted under the framework of the INSPIRE programme, funded by the Irish Government's Programme for Research in Third Level Institutions, Cycle 4, National Development Plan 2007-2013.

\section{REFERENCES}

[1] J. C. Slonczewski, "Current-driven excitation of magnetic multilayers," J. Magn. Magn. Mater., vol. L1, p. 159, 1996.

[2] M. Weisheit et al., "Electric field-induced modification of magnetism in thin-film ferromagnets," Science, vol. 315, p. 349, 2007.

[3] S. Mangin, Y. Henry, D. Ravelosona, J. A. Katine, and E. E. Fullerton, "Reducing the critical current for spin-transfer switching of perpendicularly magnetized nanomagnets," Appl. Phys. Lett., vol. 94, p. 012502, 2009.

[4] T. Maruyama et al., "Large voltage-induced magnetic anisotropy change in a few atomic layers of iron," Nature Nanotechnol., vol. 4, p. $158,2008$.

[5] Y. Shiota et al., "Voltage-assisted magnetization switching in ultrathin $\mathrm{Fe}_{80} \mathrm{Co}_{20}$ alloy layers," Appl. Phys. Expr., vol. 2, p. 06300, 2009.

[6] P. F. Carcia et al., "Perpendicular magnetic anisotropy in Pd/Co and $\mathrm{Pt} /$ Co thin-film layered structures," J. Appl. Phys., vol. 63, p. 5066, 1988.

[7] H. J. G. Draaisma, W. J. M. de Jonge, and F. J. A. den Broeder, "Magnetic interface anisotropy in $\mathrm{Pd} / \mathrm{Co}$ and $\mathrm{Pd} / \mathrm{Fe}$ multilayers," J. Magn. Magn. Mater., vol. 66, p. 351, 1987.

[8] W. B. Zeper et al., "Perpendicular magnetic anisotropy and magneto-optical Kerr effect of vapor-deposited Co/Pt-layered structures," J. Appl. Phys., vol. 65, p. 4971, 1989.

[9] C. L. Canedy, X. W. Li, and G. Xiao, "Large magnetic moment enhancement and extraordinary Hall effect in Co/Pt superlattices," Phys. Rev. B, vol. 62, p. 508, 2000.

[10] L. E. Nistor et al., "Pt/Co/oxide and oxide/Co/Pt electrodes for perpendicular magnetic tunnel junctions," Appl. Phys. Lett., vol. 94, p. 012512, 2009.

[11] A. Manchon et al., "Analysis of oxygen induced anisotropy crossover in Pt/Co/MOx trilayers," J. Appl. Phys., vol. 104, p. 043914, 2008.

[12] K. Oguz, P. Jivrajka, M. Venkatesan, G. Feng, and J. M. D. Coey, "Magnetic dead layers in sputtered $\mathrm{Co}_{40} \mathrm{Fe}_{40} \mathrm{~B}_{20}$ films," J. Appl. Phys., vol. 103, p. 07B526, 2008.

[13] G. Feng, S. van Dijken, and J. M. D. Coey, "MgO-based double barrier magnetic tunnel junctions with the thin free layers," J. Appl. Phys., vol. 105, p. 07C926, 2009.

[14] S. Cardoso et al., "Characterization of $\mathrm{CoFeB}$ electrodes for tunnel junctions," J. Appl. Phys., vol. 97, p. 10C916, 2005.

[15] Y. Jang et al., "Magnetic field sensing scheme using $\mathrm{CoFeB} / \mathrm{MgO} /$ $\mathrm{CoFeB}$ tunnelling junction with superparamagnetic CoFeB layer," Appl. Phys. Lett., vol. 8, p. 163119, 2006.

[16] S. Yuasa, Y. Suzuki, T. Katayama, and K. Ando, "Characterization of growth and crystallization processes in $\mathrm{CoFeB} / \mathrm{MgO} / \mathrm{CoFeB}$ magnetic tunnel junction structure by reflective high-energy electron diffraction," Appl. Phys. Lett., vol. 87, p. 242503, 2005. 